\title{
Dark Matter Distribution in Nearby Galaxies
}

\author{
Olivia Garrido ${ }^{1}$, Philippe Amram ${ }^{1}$, Claude Carignan ${ }^{2}$, Sébastien \\ Blais-Ouellette $^{1,2,3}$, Michel Marcelin ${ }^{1}, \&$ Delphine Russeil ${ }^{1}$ \\ ${ }^{1}$ Observatoire de Marseille, France; ${ }^{2}$ Université de Montréal, Canada; \\ ${ }^{3}$ California Institute of Technology, USA
}

\begin{abstract}
We present results obtained from a study of the mass distribution of 24 galaxies observed using Fabry-Pérot techniques, as part of the GHASP survey (see Russeil et al., this meeting). For each galaxy, we combined high resolution $\mathrm{H} \alpha$ rotation curves derived from 2-D velocity fields, with low resolution HI data, in order to determine accurately the inner slope of the rotation curve which strongly constrains the distribution of matter. Our work suggests the existence of a constant density core in the center of the dark halos.
\end{abstract}

$\Lambda \mathrm{CDM} N$-body numerical simulations predict that the innermost parts of the density profiles are cuspy: e.g., Navarro et al. $(1996 ; \mathrm{NFW})$ predict an inner slope $\alpha=-1.0$; Moore et al. (1999) an inner slope $\alpha=-1.5$. On the other hand, the observational results do not confirm these cosmological predictions but strongly suggest that dark halos have a central density core (inner slope closer to $\alpha=0$ than to $\alpha=-1$; e.g. de Blok, Bosma \& McGaugh 2003; Swaters et al. 2003; Blais-Ouellette et al. 1999, 2001, 2004). See the reviews by Bosma, and by Carignan, and the contributions of de Blok and Swaters in these proceedings.

We report here on the mass distribution for a subsample of 24 galaxies, observed for the GHASP survey, for which HI data were available from the literature. GHASP is a survey of 200 nearby galaxies observed with a FabryPérot + IPCS system (for more details about this survey, see Russeil et al. [these proceedings]; Garrido et al. 2002, 2003a, 2003b, 2004; and Amram \& Garrido 2002). For these 24 galaxies of different morphological types and luminosities, we have combined our high resolution $\mathrm{H} \alpha$ rotation curves with more extended $\mathrm{HI}$ rotation curves which can suffer, however, from beam-smearing effects. The high spatial resolution of our $\mathrm{H} \alpha$ data enable us to differentiate between the various density laws. We have compared cuspy (NFW) and core profiles (isothermal sphere).

This subsample contains both dwarf/low surface brightness galaxies (LSBs) and high surface brightness galaxies (HSBs). For LSBs, which are completely dark matter dominated, the luminous matter can essentially be ignored and the different dark matter density distributions can directly be tested from direct inversion of the rotation curve (de Blok et al. 2001). However, for HSBs, the luminous disk component cannot be ignored. Furthermore, before testing the properties of the halos using a best fit model, we first extract the dark matter component from the rotation curves. As can be seen from the left panel of Figure 1 , where we compare the $\chi^{2}$ computed from both models, the hybrid rotation 
curves are better fitted using isothermal than NFW profiles. On the right panel, the inner slope $\alpha$ of the dark halo density was a free parameter of the model; its median value is 0.15 . Both graphs suggest that the central density profiles of dark halos are better described by a core than by a cusp (Garrido 2003b).
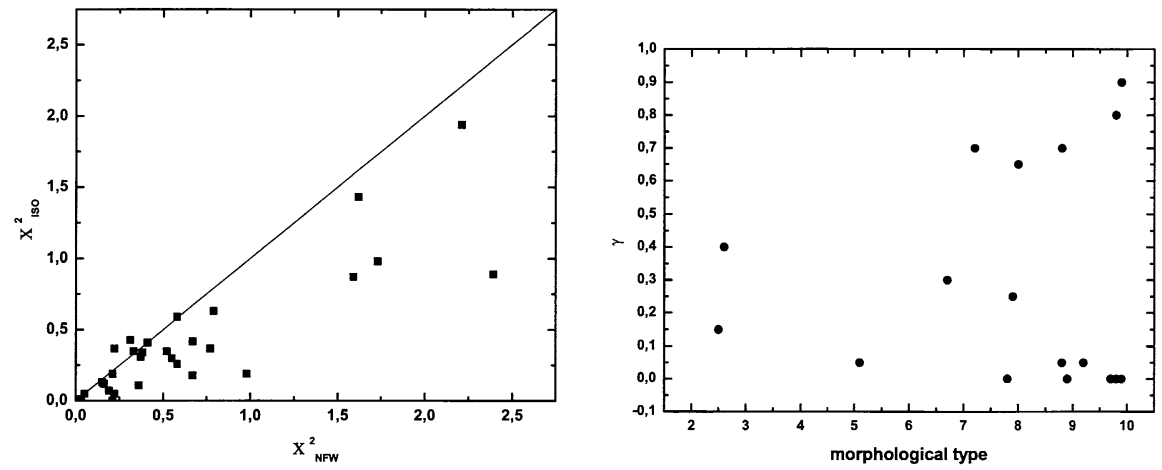

Figure 1. (left): $\chi^{2}$ obtained for the fits of our hybrid rotation curves when the isothermal sphere profile is used, versus that obtained when the NFW profile is used. (right): Inner logarithmic slope of the density profile versus morphological type.

\section{References}

Amram P., \& Garrido O. 2002, in Galaxies: the 3rd Dimension , eds. M. Rosado,

L. Binette, \& L. Arias, ASP Conf. Ser. Vol. 282, p.103

Blais-Ouellette S., Amram P., \& Carignan C. 2004, A\&A, submitted

Blais-Ouellette S., Amram P., \& Carignan C. 2001, AJ, 1221, 1952

Blais-Ouellette S., Carignan C., Amram P., \& Côté S. 1999, AJ, 120, 3027

de Blok W. J. G., Bosma A., \& McGaugh, S. 2003, MNRAS, 340, 657

de Blok W. J. G., et al. 2001, MNRAS, 340, 657

Garrido, O., et al. 2004, MNRAS, submitted

Garrido, O., et al. 2003a, A\&A, 399, 51

Garrido O. 2003b, Ph.D. Thesis, Université de Provence.

Garrido, O., Marcelin, M., Amram, P., \& Boulesteix, J. 2002, A\&A, 387, 821

Moore B., et al, 1999, MNRAS, 310, 1147

Navarro J., Frenk C., \& White S., 1996, ApJ, 462, 563

Swaters R. A., et al. 2003, ApJ, 583, 732 